\title{
Ad Hoc network traffic prediction based on the Elman neural network
}

\author{
Yuanming Ding ${ }^{1,2}$, Jiayao Gao ${ }^{2,3}$ and Xue Wang ${ }^{3}$ \\ ${ }^{1}$ Network Technology Engineering Laboratory of Smart Shipping and Logistics \\ ${ }^{2}$ Key Laboratory of Communication Networks and Information Processing \\ ${ }^{3}$ College of Information Engineering, Dalian University
}

No.10, Xuefu Street, JinZhou Xinqu, Dalian 116622, P. R. China

Key words: Traffic prediction, improved Elman neural network, Ad Hoc network

\begin{abstract}
For complex Ad Hoc network environment, based on Elman neural network prediction model, using particle swarm optimization algorithm to optimize the original Elman BP training can find the global optimization thresholds and weights of the neural network layers, and a modified Elman neural network model is proposed to predict the Ad Hoc network node traffic. According to Ad Hoc network node traffic data obtained by simulation in NS-2, network traffic predict experiment results show that the modified Elman neural network model has improved compared to the previous Elman neural network model and has a lower error and higher prediction accuracy.
\end{abstract}

\section{Introduction}

Because most of the mobile Ad Hoc network terminal is a portable computer or PDA and other mobile terminals, they often rely on battery power, transmission power is limited [1]. Therefore, the network communication efficiency is particularly important, and the network reflects the efficiency of communication is the key factor in the network traffic situation [2], the network is particularly important to study the flow conditions [3]. In Ad Hoc networks, the node traffic prediction is to improve the efficiency of network communication, network security, network intrusion prevention important guarantee [4].

In view of the network traffic prediction research mainly includes the initial steady model and is widely applied for non-stationary model [5]. Short smooth model mainly includes the related model of autoregressive model (AR), autoregressive moving average (ARMA) model, autoregressive moving average (ARIMA) model for peace, long markov process model and the related model of fractal autoregressive moving average model for peace (F - ARIMA) [6-8]. These short related model algorithm is relatively simple, but its adaptability is bad, can only describe short related characteristics of the network traffic, not in the network performance non-stationary characteristics, and increases error of prediction step also will be more and more big, short forecast period. Long related F - ARIMA model can capture long relevant characteristics of the network traffic, but the large amount of calculation, high time complexity.

Common research at present stage is a stationary model, the most representative is a neural network model, including training algorithm based on BP neural network and Elman neural network, combines the wavelet neural network, etc. [9]. It is very suitable for nonlinear prediction, neural network can describe the time varying characteristics of the network traffic, has high ability of function approximation. But the general neural network model of training and learning time is too long and high computational complexity. General Elman neural network model, the author of this paper use BP training algorithm of long time and high computational complexity, low prediction accuracy and prediction error is big, easy to fall into the most superior local defect were improved, using particle swarm optimization algorithm for threshold and weights Elman neural network is optimized, coupled with BP algorithm to forecast. Used the data in the NS - 2 simulation of Ad Hoc network traffic, then predict on the MATLAB. Based on the new Elman neural network and Elman before improvement, comparing the experimental results show that the new Elman neural network training time is shorter, less computing complexity and higher prediction accuracy, can provide reference for Ad Hoc network node traffic. 


\subsection{Elman neural network learning algorithm}

Original Elman neural network prediction model based on error back propagation algorithm (BP algorithm) as a learning and training algorithm of neural network, BP algorithm of the whole learning process is done by positive and reverse transmission, process, through which information gets spread positive from the input layer through the hidden layer, and then through the processing is completed to the opposite side of the network transmission $[10,11]$. By contrast, if the output error between the output and the theoretical value is too big, can't meet the requirements to solve the problem, will enter the forward propagation process, once upon a time in the future according to the signal transmission in the opposite direction of the error of data transmission, forward one by one, modify and threshold of each layer of neurons connected weights. Repeated iterations of this process, until the end of the last meet the requirements.

\subsection{The particle swarm optimization algorithm}

In particle swarm optimization algorithm, each particle can be considered to be optimized to solve the problem of a potential solution, a feasible solution of each particle represents the solution space. Particle swarm optimization algorithm is adopted under the type of particle operation :

$$
\begin{gathered}
V_{\mathrm{i} d}^{k+1}=V_{\mathrm{i} d}^{k}+c_{1} r_{1}\left(P_{\mathrm{i} d}^{k}-X_{\mathrm{i} d}^{k}\right)+c_{2} r_{2}\left(P_{g d}^{k}-X_{\mathrm{i} d}^{k}\right) \\
X_{\mathrm{i} d}^{k+1}=X_{\mathrm{i} d}^{k}+V_{\mathrm{i} d}^{k+1}
\end{gathered}
$$

Usually make $c_{1}=c_{2}=2.05$ and $r_{1}, r_{2}$ as a random value between $(0,1) ; X_{\text {id }}^{k}$ express particles $i$ position in the $d$ dimension $k$ times evolution iteration; $V_{\mathrm{i} d}^{k}$ express particles $i$ speed in the $d$ dimension $k$ times evolution iteration. $P_{\mathrm{i} d}^{\mathrm{k}}$ and $P_{g d}^{k}$ representative particles I individual extreme position and overall situation extreme in $d$ dimension.3. The new algorithm based on the Elman neural network model used in Ad Hoc network node traffic prediction

\section{New Elman neural network prediction Ad Hoc key node flow algorithm}

(1) The Ad Hoc node flow will be normalized processing samples, the flow of sample data values between the $(0,1)$. Normalization of the formula is:

$$
X=\frac{X_{\mathrm{i}}-X_{\min }}{X_{\max }-X_{\min }}
$$

were $X_{\mathrm{i}}$ express input values, $X_{\min }$ express minimum value of sample input, $X_{\max }$ express the maximum value of sample input, $X$ express normalized after numerical processing.

(2) For the Elman neural network connection weights and threshold of every layer is initialized, each connection weights are counted as a particle. Layers of Elman neural network connection weights and threshold of initialized initialization of particles, including the current particle swarm optimal location, best fitness of each particle, the best location, the fitness of each particle, etc.

(3) For the Elman neural network training, namely combine particle swarm algorithm of BP network connection weights parameters such as iterative optimization.

(4) Based on the current network parameters of the particle's position to calculate the fitness function value. The value of the fitness function of particle swarm algorithm error between actual output values and the theoretical output the sum of the absolute value of. Calculation formula is:

$$
F=k \sum_{i=1}^{n}\left|y_{i}-o_{i}\right|
$$

Among them, for the adjustable constant coefficient $K, i$ for the case of a node, $n$ to the total number of nodes, $o_{\mathrm{i}}$ as the actual output values, $y_{\mathrm{i}}$ as the output value of theory.

(5) Of each particle in the particle swarm to the current position and ongoing comparison of the optimal location of the optimization process, if the current position is better than that of the optimal 
location and will be updated to the current position the current optimal position of the particles. Similarly, the particle swarm's current location and comparison of the optimal location of the optimization process is, if the current position is better than that of the optimal location and will be updated to the current position of particle swarm optimal position at present. Then, according to the formula (4) (5) change the particle's position and speed, to determine whether a fitness function value satisfies the requirement of questions and, in turn, the loop iteration, until meet the conditions for network training of optimal weights and thresholds.

(6) The Elman training is complete, the output particle swarm optimal location and the corresponding optimal Elman neural network connection weights of each layer. Then, use has been training well optimized network combined with the ordinary BP algorithm to calculate the error, update weights, until meet the requirement of the problem, the simulation predictions, get the results.

\section{The results of simulation and analysis}

This section will make use of the NS - 2 simulation tool for an Ad Hoc network environment, extract the key node traffic data of the samples after the simulation, using the new Elman neural network in MATLAB data to predict, with no improved Elman neural network prediction model of predicted results were compared.

\subsection{Ad Hoc network node traffic access}

Simulation scenario according to the following Settings, the scope of scenarios for $1000 \mathrm{~m} \mathrm{x}$ $1000 \mathrm{~m}$, the network node number of 50 , maximum speed of $5 \mathrm{~m} / \mathrm{s}$, network CBR links number 100 , USES the AODV protocol, the simulation time of $168 \mathrm{~s}$, the position of the node and movement of the NS - 2 setdest random node configuration tool to configure scene. Simulations by NAM an animated demo, figure1 for a demo scene NAM a screenshot.

Based on the time interval of about 1 second in NS - 2 to extract the key node interface queue length of MAC layer, MAC layer continuous extraction node interface queue length value, as the traffic load of the sample data are stored by and as a standard of network traffic.

Through the simulation scenario simulation analysis, finally the Ad Hoc node flow is shown in Figure 2. Abscissa diagram for the time, the unit is S, y coordinate for the node flow value, unit for megabits per second. The simulation time of $168 \mathrm{~s}$, the whole simulation process for each moment node flow value, presented the node flow direction.

\subsection{Ad Hoc network node traffic prediction}

(1) Selection of input and output nodes

Elman neural network prediction model on the basis of the existing sample data of network training is to forecast. According to obtain the historical data of traffic, the selected Elman neural network's input and output nodes, which reflects the inherent law of network traffic, and achieve the purpose of flow at a certain moment in the future forecast.

In Ad Hoc networks to obtain samples of node flow, after the completion of the normalized need to determine the number of input and output nodes. The above simulation for $168 \mathrm{~s}$ of the Ad Hoc network node flow into 7 groups of data, each group contains 24 data. Traffic prediction is based on past $\mathrm{K}$ data to predict the future value of $\mathrm{X}$ moment. Experiment to node, therefore, every 2 set of 48 hour traffic data as the input vector, the output of the third group of 24 time prediction of flow at a certain moment. 


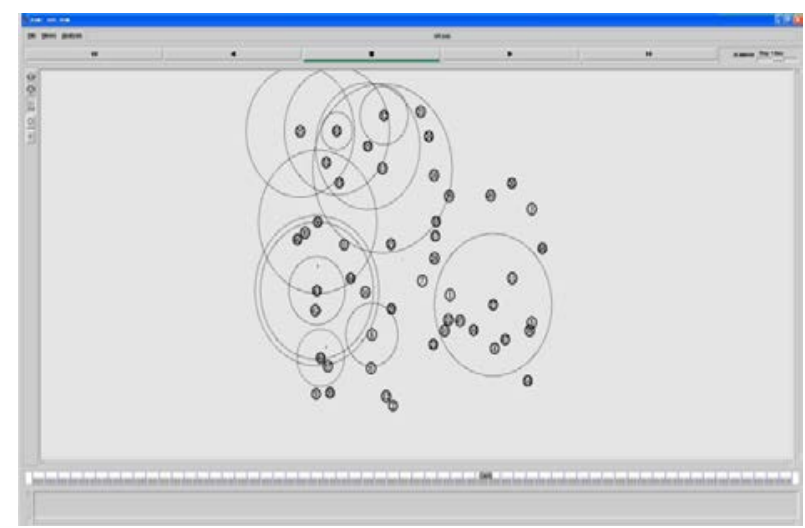

Fig.1. Experiment scene NAM cartoon picture

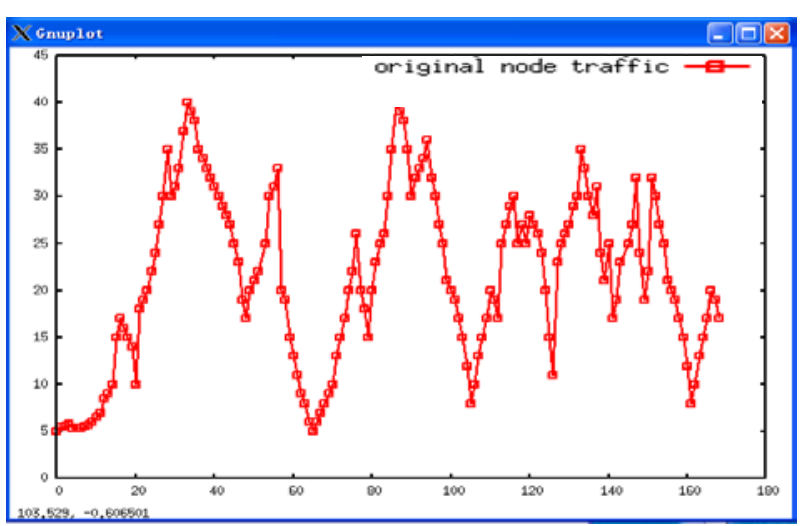

Fig.2. Ad Hoc original node traffic picture

(2) The design structure of Elman neural network model

Due to the determination of hidden layer is not yet a universal formula of ideal, can only be based on experience or training in the experiment to determine. Used to select the best hidden layer several empirical formula is as follows:

$$
\left\{\begin{array}{l}
n=\log _{2}^{m} \\
n=2 m+1 \\
n=\sqrt{m+k}+b
\end{array}\right.
$$

Among them, $\mathrm{n}$ is the number of neurons in hidden layer, $\mathrm{m}$ is the number of input neurons, $\mathrm{k}$ is the number of output neurons, $\mathrm{b}$ is the constant belong1 to10. According to the above method to determine the Elman neural network model structure for the 2-14-6-1.

(3) Using particle swarm optimization Elman connection weights and parameters.

Initialization parameters of particle swarm, set the size of the particle swarm to 10, rate of maximum and minimum value of 1 and -1 respectively, individual maximum and minimum value of 5 and -5 respectively, evolutionary iteration number is 50 times. After initialization parameter, using Newelm command in MATLAB to form Elman neural network, using $\mathrm{c}++$, input node traffic samples, complete the node flow forecast, map predicted traffic.

For comparison, the experiment is not only using the improved Elman neural network forecasting model for traffic prediction, also use the improved Elman neural network forecasting model for traffic prediction, two groups of prediction is used in NS - 2 simulation of Ad Hoc node traffic samples. Figure 3 is not improved Elman neural network prediction of flow and the original node flow diagram, Figure 4 for the improved Elman neural network to predict flow and the original node flow diagram, Figure 5 is not improved Elman and improved Elman neural network to predict traffic flow chart and the original node.

To improve before and after the two Elman neural network forecasting model the prediction error of the prediction results of the analysis of the commonly used mean absolute error (MAE), mean relative error (MAPE), mean square error (SSE), mean square error (MSE), its computation formula is as follows:

$$
\begin{gathered}
M A E=\frac{1}{n} \sum_{i=1}^{n}|y(i)-o(i)| \\
M A P E=\frac{1}{n} \sum_{i=1}^{n}\left|\frac{o(i)-y(i)}{y(i)}\right| \\
M S E=\frac{1}{n} \sqrt{\sum_{i=1}^{n}(o(i)-y(i))^{2}} \\
S S E=\sum_{\mathrm{i}=1}^{\mathrm{n}}(o(i)-y(i))^{2}
\end{gathered}
$$

$\mathrm{N}$ is the flow of the sample input data is composed of n points, $y(i)$ said the point of actual flow numerical, ${ }^{o(i)}$ represent the predicted flow value of the $\mathrm{i}$-th point. 
Table 1 .The result of four prediction deviation

\begin{tabular}{ccccc}
\hline Name & MAE & MAPE & MSE & SSE \\
\hline Unimproved Elman & 0.0673 & $18.49 \%$ & 0.0064 & 0.0640 \\
New Elman & 0.0571 & $13.37 \%$ & 0.0044 & 0.0436 \\
\hline
\end{tabular}

Experiments of the two kinds of models of several kinds of errors were analysed and calculated respectively, the results are shown in Table 1.

Analysis of various performance indicators, including the smaller mean square error and the error sum of squares, indicated that the model in Ad Hoc node traffic prediction to the data fitting degree is higher, the higher the precision. The lower the average absolute error and relative error, show that the model of Ad Hoc node traffic forecast the better the results. Through comparison and analysis to improve both before and after the error of the prediction model can be seen that the improved BP training algorithm based on particle swarm optimization combined with Elman neural network prediction model of nodes in Ad Hoc network traffic prediction in various performance indicators are better than that of before improved Elman neural network prediction model.

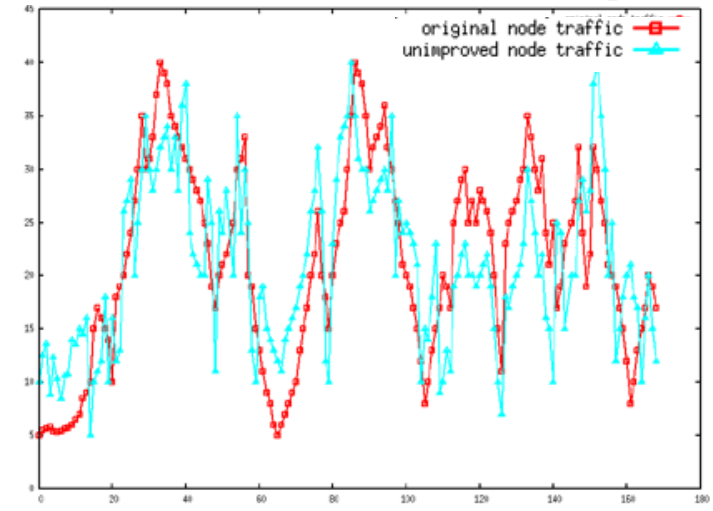

Fig.3. Unimproved Elman prediction traffic picture

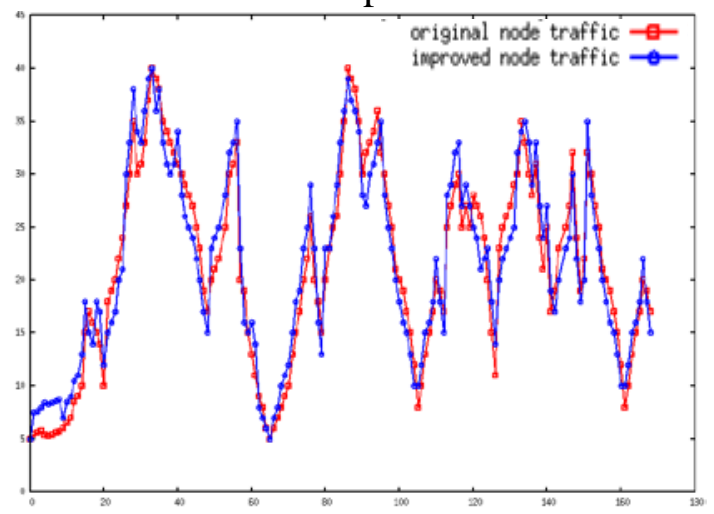

Fig.4. Improved Elman prediction traffic picture

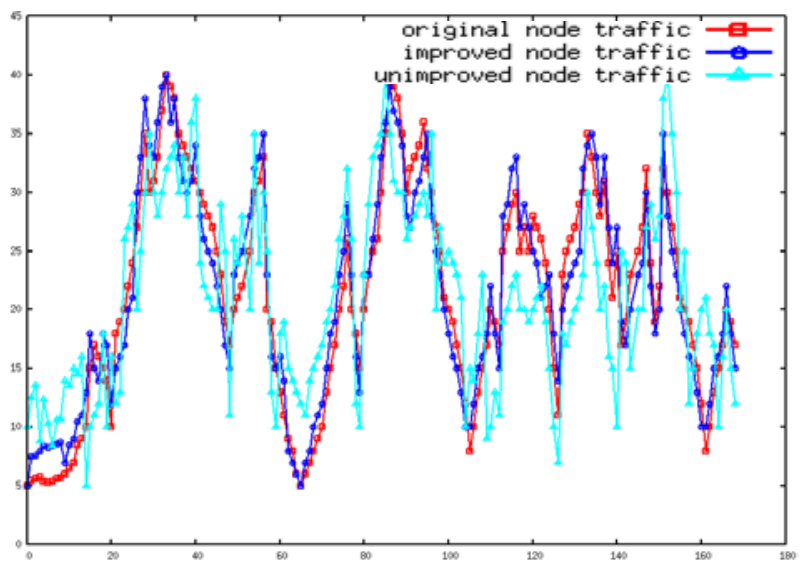

Fig.5. The two Elman prediction traffic picture

\section{The conclusion}

As a result of the Ad Hoc network environment is complicated, the improved Elman neural network prediction model is used in Ad Hoc network node traffic prediction has high prediction accuracy and lower error, can well predict the direction of node flow of Ad Hoc network communication has important significance. The improved Elman neural network prediction model can also be used in the Ad Hoc network intrusion detection, according to the changes in the flow to judge whether the invasion, the Ad Hoc network security and defense study has certain reference 
significance.

\section{References}

[1] Yemeni, R. and Sarje, A. K., "Secure AODV protocol to mitigate black hole attack in mobile Ad Hoc networks," Proc. ICCCN, 1-5 (2012).

[2] Roopak, M. and Reddy, B., "Black hole attack implementation in AODV routing protocol," International Journal of Scientific \& Engineering Research. Papers 4(5), 402-406 (2013).

[3] Yeats., [Wireless network attack and defense principle and practice] Tsinghua university press, Beijing (2012).

[4] Cochenour, B., Mullen, L. and Muth, J., "Effect of scattering albedo on attenuation and polarization of light underwater," Optics Letters. Papers 35(12), 2088-2090 (2010).

[5] Zhang, X., [Ad Hoc network traffic self-similarity research] Shanghai jiaotong university press, Shanghai (2010).

[6] Marti, S., Giuli, T.J. and Lai, K., "Mitigating routing misbehavior in mobile Ad Hoc networks," Proc. the 6th Annual Intl. Conf. on Mobile Computing and Networking, 255-265 (2010).

[7] Cabrera, J. B. D., Gutierrez, C. and Mehra, R. K., "Ensemble methods for anomaly detect- ion and distributed intrusion detection in mobile Ad Hoc networks," Information Fusion. Papers 9, 96-119 (2008).

[8] Shivashankar, T., Sivakumar, B., Varaprasad, M.,"Identification of critical node for the efficient performance in MANET," International Journal of Advanced Computer Science and Applications. Papers 3(1), 166-171 (2012).

[9] Callaw, P., Newman, B. K., Strogatez, R., "Network robustness and fragility: Percolation on random graphs," Phys Rev. Lett. Papers 85(25), 5468-5741 (2010).

[10] Tong X., Cui M. and Ma C., "The research of distributed intrusion detection and communication protocol based on entity," Proc. WiCOM, 1-4 (2008).

[11] Yi P., Zou F. T. and Zou Y., "Performance analysis of mobile ad hoc network sunder flooding attacks," Journal of Systems Engineering and Electronics. Papers 22(2), 334-339 (2010). 